\title{
NOTIZEN
}

\section{Quantenmechanik in Separations-Koordinaten}

\section{Ernst Bretnütz}

Institut für Theoretische Physik A der Technischen Hochschule Braunschweig

(Z. Naturforschg. 20 a, 485-486 [1965]; eingegangen am 19. Januar 1965)

If the Schröninger equation can be solved by a product of functions, which depend only on one variable (separation), then a complete set of commutable operators exists, which forms a set of quantum mechanical integrals of motion. The eigenvalues of these operators are the separation constants. The operators are explicitely formulated; the physical meaning is discussed.

SтÄCKEL entwickelte erstmalig eine systematische Theorie für die Separation der Hamilton-Jacobischen Differentialgleichung ${ }^{1}$, die später von Robertson ${ }^{2}$ mit den gleichen Begriffsbildungen auf die Separation der zeitunabhängigen SchröDINGER-Gleichung übertragen wurde ${ }^{3}$. Notwendig und hinreichend für eine Separation beider Differentialgleichungen in krummlinigen, orthogonalen Koordinaten $\left(x_{1}, \ldots, x_{f} ; f\right.$ ist die Zahl der Freiheitsgrade des Systems) ist die Existenz der „SтӓсKEL-Determinante“

$$
\left|\varphi_{i j}\left(x_{i}\right)\right| \quad(i, j=1, \ldots, f),
$$

deren Elemente Funktionen nur der einen Koordinate $x_{i}$ sind. Für eine Separation der Schrödinger-Gleichung lassen sie sich folgendermaßen aus dem Linienelement

$$
\mathrm{d} s^{2} \underset{\overline{\text { Def }}}{\sum_{i=1}^{f}} H_{i}^{2} \mathrm{~d} x_{i}^{2}
$$

des betreffenden Koordinatensystems bestimmen: Mit

$$
H \underset{\text { Def. }}{\stackrel{f}{i=1}} H_{i}
$$

gestatten separable Koordinaten eine Zerlegung des Quotienten

$$
H / H_{i}{ }^{2}=f_{i}\left(x_{i}\right) \cdot a_{i}(x \neq i) \quad(i=1, \ldots, f)
$$

in ein Produkt von zwei Funktionen, von denen $f_{i}\left(x_{i}\right)$ nur von der Koordinate $x_{i}, a_{i}(x \neq i)$ jedoch von allen übrigen $x_{j}(j \neq i)$ abhängt. Dann ist durch

$$
\varphi=H / \prod_{i=1}^{f} f_{i}
$$

der Wert der Determinante $\varphi=\left|\varphi_{i j}\right|$ bestimmt. Ersichtlich ist, abgesehen von singulären Punkten, $\varphi \neq 0$. Die algebraischen Komplemente der ersten Spalte von $\varphi$ sind weiterhin durch

$$
\Phi_{j 1}=\varphi / H_{j}{ }^{2} \quad(j=1, \ldots, f)
$$

1 P. SтёскеL, Habilitationsschrift, Halle/S. 1891; vgl. auch Math. Ann. 42, 537 [1893], Abschn. 2.

2 H. P. Robertson, Math. Ann. 98, 749 [1928]. gegeben. Dies reicht aus, um $\varphi$ für ein bestimmtes Separationskoordinatensystem aufzustellen, wenn man noch beachtet, daß die Elemente der $i$-ten Zeile nur von $x_{i}$ abhängen.

Die potentielle Energie $U\left(x_{1}, \ldots, x_{f}\right)$ muß sich im separablen Fall in der Form

$$
U(x)=\sum_{j=1}^{f} \frac{\Phi_{i 1}}{\varphi} \mu_{i}\left(x_{i}\right)=\sum_{i=1}^{f} \frac{\mu_{i}\left(x_{i}\right)}{H_{i}^{2}}
$$

mit beliebigen Funktionen $\mu_{i}\left(x_{i}\right)$ von nur einer Veränderlichen $x_{i}$ darstellen lassen, um die Separation nicht zu stören.

Die zeitunabhängige Schrödrnger-Gleichung in orthogonalen Koordinaten

$$
\begin{array}{r}
\sum_{i=1}^{t} \frac{1}{H} \frac{\partial}{\partial x_{i}}\left(\frac{H}{H_{i}^{2}} \frac{\partial \psi}{\partial x_{i}}\right)+k^{2}(E-U(x)) \psi=0 \\
\left(k^{2}=2 m / \hbar^{2}\right)
\end{array}
$$

lautet in Separationskoordinaten mit dem Ansatz

$$
\psi\left(x_{1}, \ldots, x_{f}\right)=\prod_{i=1}^{f} X_{i}\left(x_{i}\right),
$$

worin $X_{i}\left(x_{i}\right)$ nur von $x_{i}$ abhängt:

$$
\sum_{i=1}^{f} \frac{1}{H_{i}^{2}} \frac{1}{f_{i} X_{i}} \frac{\mathrm{d}}{\mathrm{d} x_{i}}\left(f_{i} \frac{\mathrm{d} X_{i}}{\mathrm{~d} x_{i}}\right)+k^{2}(E-U(x))=0
$$

oder mit der Abkürzung

$$
s_{i}\left(x_{i}\right)=\frac{1}{f_{i} X_{i}} \frac{\mathrm{d}}{\mathrm{d} x_{i}}\left(f_{i} \frac{\mathrm{d} X_{i}}{\mathrm{~d} x_{i}}\right)
$$

und mit dem obigen Ansatz für $U(x)$

$$
\sum_{i=1}^{f} \Phi_{i 1}\left[s_{i}\left(x_{i}\right)-k^{2} \mu_{i}\left(x_{i}\right)+k^{2} \alpha_{1} \varphi_{i 1}\right]=0 . \quad\left(\alpha_{1} \equiv E\right)
$$

Diese Beziehung zusammen mit den $f-1$ weiteren Beziehungen

$$
\sum_{i=1}^{t} \Phi_{i 1} \varphi_{i j}=0 \quad(j=2,3, \ldots, f)
$$

führt zu den $f$ Separationsgleichungen in Form von gewöhnlichen Differentialgleichungen, die durch die Separationskonstanten $\alpha_{j}$ verbunden sind :

$$
\begin{aligned}
& s_{i}\left(x_{i}\right)-k^{2} \mu_{i}\left(x_{i}\right)+k^{2} \sum_{i=1}^{f} \alpha_{j} \varphi_{i j}\left(x_{i}\right)=0 \\
&(i=1,2, \ldots, f) .
\end{aligned}
$$

Da die Determinante $\varphi=\left|\varphi_{i j}\right|$ von Null verschieden vorausgesetzt wurde, lassen sich die Separationsglei-

\footnotetext{
3 siehe auch P. M. Morse u. H. Feshbach, Methods of Theoretical Physics, McGraw-Hill Book Co., Inc., New York, Toronto, London 1953, Bd. I, S. 508, 655.
} 
chungen nach den Separationskonstanten auflösen. Hierzu wird die $i$-te Gleichung mit $\Phi_{i l}$ multipliziert und anschließend über $i$ summiert :

oder

$$
\begin{aligned}
& \sum_{i=1}^{f} \Phi_{i l}\left(s_{i}-k^{2} \mu_{i}\right)+k^{2} \varphi \alpha_{l}=0 \\
& \alpha_{l}=\sum_{i=1}^{f} \frac{\Phi_{i l}}{\varphi}\left(-\frac{1}{k^{2}} s_{i}\left(x_{i}\right)+\mu_{i}\left(x_{i}\right)\right.
\end{aligned}
$$

$$
\text { (vgl. } \left.{ }^{4}\right) \quad(l=1,2, \ldots, f) .
$$

Diese Funktionen können als Bewegungsintegrale aufgefaßt und entsprechend quantenmechanisch gedeutet werden:

$$
\alpha_{l}=\sum_{i=1}^{f} \frac{\Phi_{i l}}{\varphi}\left[-\frac{\hbar^{2}}{2 m} \frac{1}{f_{i} X_{i}} \frac{\mathrm{d}}{\mathrm{d} x_{i}}\left(f_{i} \frac{\mathrm{d} X_{i}}{\mathrm{~d} x_{i}}\right)+\mu_{i}\left(x_{i}\right)\right] .
$$

Wird dies mit $\quad \psi=\prod_{i=1}^{t} X_{i}\left(x_{i}\right) \quad$ multipliziert,

$$
\alpha_{l} \psi=\sum_{i=1}^{f} \Phi_{i l}\left[-\frac{\hbar^{2}}{2 m} \frac{1}{\varphi f_{i}} \frac{\partial}{\partial x_{i}}\left(f_{i} \frac{\partial \psi}{\partial x_{i}}\right)+\frac{\mu_{i}}{\varphi} \psi\right],
$$

so liegt es nahe, entsprechend der Definition der Impulsoperatoren in krummlinigen Koordinaten für skalare Wellenfunktionen ${ }^{5}$

$$
p_{l} \psi=\frac{\hbar}{i} \frac{1}{\sqrt[4]{g}} \frac{\partial}{\partial x_{l}}(\sqrt[4]{g} \psi),
$$

( $g$ Determinante des kovarianten Maßtensors) mit den Operatoren

$$
\left.p_{l} \psi=\frac{\hbar}{i} \frac{1}{\sqrt{\varphi f_{l}}} \frac{\partial}{\partial x_{l}}\left(\sqrt{\varphi f_{l}} \psi\right) \quad \text { (es ist } g=H^{2}\right)
$$

$\alpha_{l} \psi$ als Operatorenfunktionen zu schreiben:

$$
\alpha_{l} \psi=\sum_{i=1}^{f} \Phi_{i l}\left[\frac{1}{2 m} \frac{1}{\sqrt{\varphi f_{i}}} p_{i} f_{i} p_{i} \frac{1}{\sqrt{\varphi} \bar{f}_{i}}+\frac{\mu_{i}}{\varphi}\right] \psi .
$$

$\Phi_{i l}$ braucht nicht in den Symmetrisationsprozeß einbezogen zu werden, da es nicht von $x_{i}$ abhängt und demzufolge mit den $p_{i}$ vertauschbar ist. Der symmetrische Aufbau der Produkte läßt sofort erkennen, daß die $\alpha_{l}$ selbstadjungierte Operatoren sind. Sie sind weiterhin paarweise miteinander vertauschbar:

Mit der Abkürzung

wird

$$
\begin{aligned}
& t_{i}=\frac{1}{2 m} \frac{1}{\sqrt{f_{i}}} p_{i} f_{i} p_{i} \frac{1}{\sqrt{f_{i}}}+\mu_{i} \\
& \alpha_{l} \psi=\sum_{i=1}^{f} \Phi_{i l} \frac{1}{\sqrt{\varphi}} t_{i} \frac{1}{\sqrt{\varphi}} \psi .
\end{aligned}
$$

Damit kann der Kommutator geschrieben werden:

$$
\begin{aligned}
{\left[\alpha_{\varkappa}, \alpha_{\lambda}\right] } & =\sum_{m, n} \frac{\Phi_{m \varkappa}}{\sqrt{\varphi}} t_{m} \frac{\Phi_{n \lambda}}{\varphi} t_{n} \frac{1}{\sqrt{\varphi}} \\
& -\sum_{m, n} \frac{\Phi_{n \hat{\lambda}}}{\sqrt{\varphi}} t_{n} \frac{\Phi_{m \varkappa}}{\varphi} t_{m} \frac{1}{\sqrt{\varphi}} .
\end{aligned}
$$

${ }^{4}$ Für die Hamilton-Jacobische Differentialgleichung sind die $f$ quadratischen ersten Integrale von STÄcKEL angegeben worden: C. R. Acad. Sci., Paris 116, 485 [1893].
Wird hierin $n$ und $m$ vertauscht

$$
\begin{aligned}
{\left[\alpha_{\varkappa}, \alpha_{\lambda}\right] } & =\sum_{m, n} \frac{\Phi_{n \varkappa}}{\sqrt{\varphi}} t_{n} \frac{\Phi_{m \lambda}}{\varphi} t_{m} \frac{1}{\sqrt{\varphi}} \\
& -\sum_{m, n} \frac{\Phi_{m \lambda}}{\sqrt{\varphi}} t_{m} \frac{\Phi_{n \varkappa}}{\varphi} t_{n} \frac{1}{\sqrt{\varphi}}
\end{aligned}
$$

und beides addiert, so ergibt sich

$$
\begin{aligned}
2\left[\alpha_{\varkappa}, \alpha_{\lambda}\right] & =\sum_{m, n} \frac{1}{\sqrt{\varphi}} t_{m} \frac{\Phi_{m \varkappa} \Phi_{n \lambda}-\Phi_{m \lambda} \Phi_{n \varkappa}}{\varphi} t_{n} \frac{1}{V \varphi} \\
& -\sum_{m, n} \frac{1}{\sqrt{\varphi}} t_{n} \frac{\Phi_{m \varkappa} \Phi_{n \lambda}-\Phi_{m \lambda} \Phi_{n \varkappa}}{\varphi} t_{m} \frac{1}{\sqrt{ } \varphi} .
\end{aligned}
$$

Nach einem Satz der Determinantentheorie (siehe etwa Kowalewski $^{6}$ ) ist der zweireihige Minor

$$
\Phi_{m \varkappa} \Phi_{n \lambda}-\Phi_{m \lambda} \Phi_{n \varkappa}
$$

der zu $\varphi$ reziproken Determinante gleich dem algebraischen Komplement zu dem gleichindizierten zweireihigen Minor in $\varphi$, multipliziert mit $\varphi$. Da aber dieses algebraische Komplement in $\varphi$ keine Elemente aus der $m$-ten und $n$-ten Zeile enthält und der Faktor $\varphi$ sich weghebt, ist der mittlere Bruchstrich mit den Operatoren $t_{m}, t_{n}$ vertauschbar. Die $t_{m}$ bzw. $t_{n}$ wirken jedoch nur auf $x_{m}$ bzw. $x_{n}$, sind also auch untereinander vertauschbar. Mithin ist

$$
\left[\alpha_{\varkappa}, \alpha_{\lambda}\right]=0 \text {. }
$$

Die ursprünglichen Beziehungen

$$
\begin{aligned}
\alpha_{l} \psi=\sum_{i=1}^{f} \Phi_{i l}\left[\frac{1}{2 m} \frac{1}{\sqrt{\varphi f_{i}}} p_{i} f_{i} p_{i} \frac{1}{\sqrt{\varphi f_{i}}}+\frac{\mu_{i}}{\varphi}\right] \psi \\
(l=1,2, \ldots, f)
\end{aligned}
$$

können also als Eigenwertgleichungen für einen voll. ständigen Satz vertauschbarer Operatoren angesehen werden, die sämtlich Konstanten der Bewegung sind, denn für $l=1$ ergibt sich der Hamilton-Operator $H$. mit dem also alle anderen Operatoren vertauschbar sind. Da andererseits die Eigenwerte $\alpha_{l}$ dieser Operatoren ursprünglich als Separationskonstanten für die Separation der SchröDinger-Gleichung auftraten, besteht die quantenmechanische Bedeutung der Separation in einer simultanen Lösung eines Eigenwertproblems für einen vollständigen Satz vertauschbarer Operatoren. Da wir oben

$$
\Phi_{i 1} \neq 0 \quad(i=1,2, \ldots, f)
$$

fordern mußten, diese Forderung jedoch für

$$
\Phi_{i k} \quad(k=2,3, \ldots, f)
$$

nicht erfüllt zu sein braucht, stellt die SchrödingerGleichung als Eigenwertgleichung des Hamilton-Operators auch die stärksten Forderungen an die gemeinsamen Eigenfunktioen; diese werden also durch die Schrödinger-Gleichung bereits vollständig definiert.

Herrn Prof. Dr. M. KoHler danke ich für die Anregung zu dieser Arbeit und für wertvolle weiterführende Hinweise.

5 Siehe etwa W. Pauli im Handbuch der Physik, herausgeg. v. S. FlüGGE, Verlag Springer, Berlin, Göttingen, Heidelberg 1958, Bd. V, Teil 1, S. 40.

${ }^{6} \mathrm{G}$. Kowalewski, Einführung in die Determinantentheorie, Verlag de Gruyter, 4. Auflage, Berlin 1954, S. 80. 
auf den weit unter der Nachweisbarkeitsgrenze liegenden Amplitudenkontrast

$$
\frac{\Delta j}{j}(\mathrm{Am}) \approx \frac{\Delta x}{x_{\mathrm{K}}}=10^{-3} .
$$

Die im betrachteten Fall mehr als 20-fache Verstärkung durch Phasenkontrast bildet also die relativen Dichteschwankungen $\Delta x / x$ von $3,6 \%$ mit einem Kontrast von 78\% des Amplitudenkontrastes der ganzen Folie $\left(x / x_{\mathrm{K}}=3 \cdot 10^{-2}\right) \mathrm{ab}$ !

(Zur Bedeutung der Bezeichnungen Phasenkontrast und Amplitudenkontrast siehe Lenz ${ }^{10}$.)

Von Ruska (briefl. Mitt.) beobachtete kleine Verschiebungen der "Granula" beim Durchfokussieren sind durch die von $\Delta z$ abhängige Zuordnung der statistischen Schwankungen $\mathrm{zu}$ den Auswahlbereichen $\Lambda^{2} \pi / 4$ erklärlich. Das von van Dorsten (Vortrag Bonn,

10 F. Lenz, 4. Intern. Kongr. Elektronenmikroskopie, Berlin 1958, Verh. Bd. 1, S. 306, Springer 1960.
9. 11. 64) berichtete Verschwinden und Neuentstehen solcher Granula kann außer durch Fokusverschiebungen und örtliche, zeitlich schwankende Aufladungen auch durch Platzwechselvorgänge, die die statistische Dichteverteilung örtlich verändern, gedeutet werden.

Wie schon oben angedeutet, ist (5) eine nur für große $|\Delta z|$ gültige Näherung. Für kleine $|\Delta z|$, d. h. große Aperturen $\Lambda / \Delta z$ verkürzt sich der optische Weg des gebeugten Strahls durch die öffnungsfehlerbedingte Wellenaberration nennenswert, so daß die Fläche des hervorgehobenen Bereichs für $|\Delta z| \rightarrow 0$ einem endlichen Wert zustrebt ${ }^{6}$. Außerdem verliert die Überlegung von Lenz und Scheffels ${ }^{2}$ natürlich ihren Sinn, wenn $A / \Delta z$ größer als die durch eine materielle Blende begrenzte Objektivapertur wird. Deshalb nimmt der Phasenkontrast, wie auf Abb. 8 in ${ }^{1}$ ersichtlich, für kleine Defokussierungen mit dieser ab.

Ich danke Herrn Prof. Dr. F. Lenz, Tübingen, für die freundliche Durchsicht des Manuskriptes.

\section{ESR-Spektren des Benzyl- und des $\alpha$-Hydroxybenzylradikals}

\author{
Hanns Fischer
}

Deutsches Kunststoff-Institut, Darmstadt

(Z. Naturforschg. 20 a, 488-489 [1965]; eingegangen am 20. Februar 1965)

Hochaufgelöste Elektronenspinresonanz(ESR)-Spektren des Benzylradikals (I) sind kürzlich von DixoN und Norman ${ }^{1}$ während der Reaktion von Toluol mit Hydroxylradikalen beobachtet worden. Wir haben dieses Radikal und sein Derivat, das $\alpha$-Hydroxybenzylradikal (II), bei der Reaktion von Hydroxylradikalen mit Phenylessigsäure bzw. Benzylalkohol erhalten. Dabei wurden einige Besonderheiten der Radikalreaktionen und der ESR-Spektren von I und II festgestellt.<smiles>[3H]C1CCC(C=O)C1</smiles><smiles>CC1CCC(CO)C1</smiles>

Die Untersuchungen wurden mit der von Dixon und Norman ${ }^{2}$ und auch von uns ${ }^{3}$ bereits früher ausführlich beschriebenen Durchflußtechnik durchgeführt.

Bei der Reaktion von Phenylessigsäure $\left(\mathrm{C}_{6} \mathrm{H}_{5}-\right.$ $\mathrm{CH}_{2}-\mathrm{COOH}$ ) in $10^{-1}$-mol. wäßriger Lösung mit $\mathrm{Hy}$ droxylradikalen werden ESR-Spektren beobachtet, deren Struktur von der Konzentration der Schwefelsäure in den Lösungen abhängt. Bei niedriger Säurekonzen-

1 W. T. Dixon u. R. O. C. Norman, J. Chem. Soc. 1964, 4857. tration $\left(c<10^{-2} \mathrm{Mol} / l\right)$ zeigen die Spektren eine Gesamtausdehnung von 75 Oe und besitzen neben kleineren, nicht völlig aufgelösten Aufspaltungen eine Dublettaufspaltung von $a_{\mathrm{D}}=(34 \pm 1)$ Oe. Sie sind deshalb mit großer Wahrscheinlichkeit auf Radikale vom Cyclohexadienyltyp zurückzuführen, die durch Anlagerung von Hydroxylradikalen an die Benzolringe der Phenylessigsäuremolekeln entstehen. Bei höherer Säurekonzentration verschwinden die Spektren mit der Dublettstruktur. Es erscheint das in Abb. 1 wiedergegebene Spektrum, das nach seiner Linienstruktur dem Benzylradikal (I) zugeordnet werden muß.

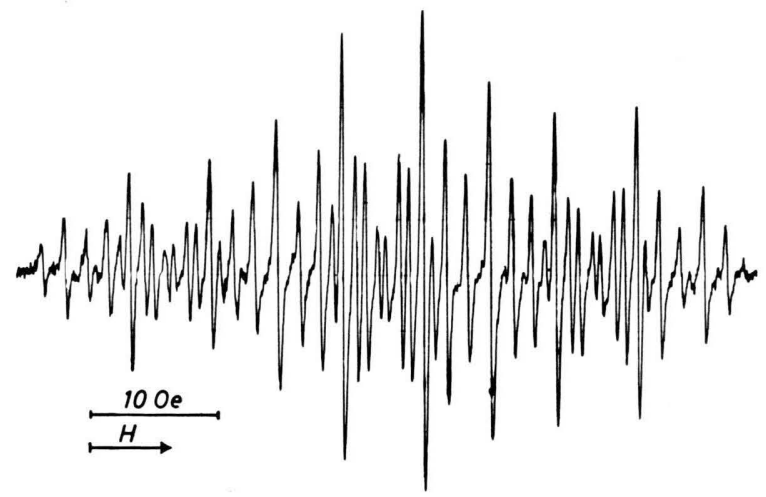

Abb. 1. ESR-Spektrum des Benzylradikals.

Aus der Analyse dieses Spektrums ergeben sich die in Tab. 1 angegebenenen Aufspaltungsparameter.

Bei der Reaktion von Benzylalkohol $\left(\mathrm{C}_{6} \mathrm{H}_{5}-\mathrm{CH}_{2} \mathrm{OH}\right)$ mit Hydroxylradikalen treten bei niedriger Säurekonzentration ebenfalls Radikale vom Cyclohexadienyltyp

2 W. T. Dixon u. R. O. C. Norman, J. Chem. Soc. 1963, 3119.

${ }^{3}$ H. Fischer, Z. Naturforschg. 19 a, 866 [1964]. 